\title{
Analytic study of self-gravitating polytropic spheres with light rings
}

\author{
Shahar Hod ${ }^{1,2, a}$ \\ ${ }^{1}$ The Ruppin Academic Center, Emeq Hefer 40250, Israel \\ 2 The Hadassah Academic College, Jerusalem 91010, Israel
}

Received: 23 March 2018 / Accepted: 18 May 2018 / Published online: 26 May 2018

(C) The Author(s) 2018

\begin{abstract}
Ultra-compact objects describe horizonless solutions of the Einstein field equations which, like black-hole spacetimes, possess null circular geodesics (closed light rings). We study analytically the physical properties of spherically symmetric ultra-compact isotropic fluid spheres with a polytropic equation of state. It is shown that these spatially regular horizonless spacetimes are generally characterized by two light rings $\left\{r_{\gamma}^{\text {inner }}, r_{\gamma}^{\text {outer }}\right\}$ with the property $\mathcal{C}\left(r_{\gamma}^{\text {inner }}\right) \leq$ $\mathcal{C}\left(r_{\gamma}^{\text {outer }}\right)$, where $\mathcal{C} \equiv m(r) / r$ is the dimensionless compactness parameter of the self-gravitating matter configurations. In particular, we prove that, while black-hole spacetimes are characterized by the lower bound $\mathcal{C}\left(r_{\gamma}^{\text {inner }}\right) \geq 1 / 3$, horizonless ultra-compact objects may be characterized by the opposite dimensionless relation $\mathcal{C}\left(r_{\gamma}^{\text {inner }}\right) \leq 1 / 4$. Our results provide a simple analytical explanation for the interesting numerical results that have recently presented by Novotný et al. (Phys Rev D 95:043009, 2017).
\end{abstract}

\section{Introduction}

Curved spacetimes describing highly compact astrophysical objects may be characterized, according to the Einstein field equations, by null circular geodesics (closed light rings) [1$3]$ on which photons and gravitons can orbit the central selfgravitating compact object. These null orbits are interesting from both a theoretical and an astrophysical points of view and their physical properties have been studied extensively by physicists and mathematicians during the last five decades (see [1-22] and references therein).

As demonstrated in $[4,5]$, the optical appearance of a highly compact collapsing star is determined by the physical properties of its null circular geodesic $[4,5]$. Likewise, the intriguing phenomenon of strong gravitational lensing by highly compact objects is related to the presence of light rings

\footnotetext{
a e-mail: shaharhod@gmail.com
}

in the corresponding curved spacetimes [6]. In addition, as explicitly shown in [7-17], the discrete quasinormal resonant spectra of compact astrophysical objects are related, in the eikonal limit, to the physical properties (the circulation time and the characteristic instability time scale) of the null circular geodesics that characterize the corresponding curved spacetimes ${ }^{1}[23,24]$.

Interestingly, it has recently been proved that spherically symmetric black-hole spacetimes must posses at least one light ring [25]. In particular, the theorem presented in [25] has revealed the fact that the innermost null circular geodesic of an asymptotically flat black hole must be located in a highly compact spacetime region which is characterized by the dimensionless lower bound $[25]^{2}$

$\frac{m\left(r_{\gamma}^{\text {in }}\right)}{r_{\gamma}^{\text {in }}} \geq \frac{1}{3}$ for black holes

where $r_{\gamma}^{\text {in }}$ and $m\left(r_{\gamma}^{\text {in }}\right)$ are respectively the innermost (smallest) radius of the light ring which characterizes the black-hole spacetime and the total gravitational mass contained within this sphere.

Ultra-compact objects, spatially regular horizonless matter configurations which, like black-hole spacetimes, possess light rings, have attracted much attention in recent years as possible exotic alternatives to the canonical black-hole spacetimes [26-35]. In particular, in a very interesting work, Novotný, Hladík, and Stuchlík [35] (see also [24]) have recently studied numerically the physical properties of spherically symmetric self-gravitating isotropic fluid spheres with

\footnotetext{
${ }^{1}$ It is worth mentioning that, as shown in [23], there are some nontrivial situations in which this relation is violated. However, as shown in [24], this relation holds true for the trapping polytropic spheres that we shall consider in the present paper.

${ }^{2}$ We shall use natural units in which $G=c=1$.
} 
a polytropic pressure-density equation of state of the form [36]

$$
p(\rho)=k_{\mathrm{p}} \rho^{1+1 / n},
$$

where the dimensionless physical parameter $n$ is the polytropic index of the fluid system [36]. It is worth emphasizing that the self-gravitating ultra-compact trapping polytropic spheres were first mentioned in [37].

Interestingly, it has been explicitly demonstrated numerically in [35] that spatially regular polytropic spheres may possess two light rings $\left\{r_{\gamma}^{\text {in }}, r_{\gamma}^{\text {out }}\right\}$ (see [26,33] for related discussions) which are characterized by the compactness inequality

$\frac{m\left(r_{\gamma}^{\text {in }}\right)}{r_{\gamma}^{\text {in }}} \leq \frac{m\left(r_{\gamma}^{\text {out }}\right)}{r_{\gamma}^{\text {out }}}$

In particular, the intriguing fact has been revealed in [35] that the spherically symmetric self-gravitating horizonless polytropic spheres may be characterized by closed light rings (null circular geodesics) with the remarkably small compactness relation

$\frac{m\left(r_{\gamma}^{\text {in }}\right)}{r_{\gamma}^{\text {in }}}<\frac{1}{3} \quad$ for horizonless polytropic spheres.

It is worth emphasizing the fact that the dimensionless relation (4), observed numerically in [35] for the horizonless selfgravitating polytropic spheres, violates the lower bound (1) which, as explicitly proved in [25], characterizes the innermost light rings of spherically symmetric black-hole spacetimes.

The main goal of the present paper is to study analytically the physical and mathematical properties of the horizonless ultra-compact polytropic matter configurations. In particular, below we shall provide compact analytical proofs for the characteristic intriguing relations (3) and (4) that have recently been observed numerically in [35] for the spherically symmetric spatially regular isotropic fluid stars.

\section{Description of the system}

Following the interesting physical model studied numerically in [35], we shall consider asymptotically flat isotropic matter configurations which are characterized by the spherically symmetric static line element $[2]^{3}$

$d s^{2}=-e^{-2 \delta} \mu d t^{2}+\mu^{-1} d r^{2}+r^{2}\left(d \theta^{2}+\sin ^{2} \theta d \phi^{2}\right)$,

\footnotetext{
3 Here $(t, r, \theta, \phi)$ are the familiar Schwarzschild spacetime coordinates.
}

where $\delta=\delta(r)$ and $\mu=\mu(r)$. Spatially regular matter configurations are characterized by the functional behaviors $[18,19]$

$\mu(r \rightarrow 0)=1+O\left(r^{2}\right)$ and $\delta(0)<\infty$,

in the near-origin $r \rightarrow 0$ limit. In addition, asymptotically flat regular spacetimes are characterized by the simple large- $r$ functional relations $[18,19,38]$

$\mu(r \rightarrow \infty) \rightarrow 1 \quad$ and $\quad \delta(r \rightarrow \infty) \rightarrow 0$.

The non-linearly coupled Einstein-matter field equations, $G_{v}^{\mu}=8 \pi T_{v}^{\mu}$, can be expressed by the differential relations $[18,19]^{4}$

$\mu^{\prime}=-8 \pi r \rho+\frac{1-\mu}{r}$,

and

$\delta^{\prime}=-\frac{4 \pi r(\rho+p)}{\mu}$,

where the radially-dependent density and pressure functions

$\rho \equiv-T_{t}^{t} \quad$ and $\quad p \equiv T_{r}^{r}=T_{\theta}^{\theta}=T_{\phi}^{\phi}$,

denote the components of the isotropic energy-momentum tensor [39]. We shall assume that the spherically symmetric asymptotically flat matter configurations respect the dominant energy condition [40]

$0 \leq|p| \leq \rho$.

From the Einstein Eqs. (8) and (9) and the conservation relation

$T_{r ; \mu}^{\mu}=0$,

one can derive the characteristic compact differential equation

$P^{\prime}(r)=\frac{r}{2 \mu}[\mathcal{R}(\rho+p)+2 \mu(-\rho+p)]$,

for the gradient of the radially-dependent isotropic pressure function

$P(r) \equiv r^{2} p(r)$,

\footnotetext{
${ }^{4}$ Here a prime ${ }^{\prime}$ denotes a spatial derivative with respect to the radial coordinate $r$.
} 
where

$\mathcal{R}(r) \equiv 3 \mu-1-8 \pi r^{2} p$.

Below we shall analyze the spatial behavior of the characteristic dimensionless compactness function

$\mathcal{C}(r) \equiv \frac{m(r)}{r}$,

where the mass $m(r)$ of the matter fields contained within a sphere of radius $r$ is given by the simple integral relation $[18,19]$

$m(r)=4 \pi \int_{0}^{r} x^{2} \rho(x) d x$.

Taking cognizance of Eqs. (8) and (17), one deduces the simple dimensionless functional relation

$\mu(r)=1-\frac{2 m(r)}{r}$.

For later purposes we note that asymptotically flat regular matter configurations are characterized by the asymptotic radial behavior $[18,19]$

$r^{3} p(r) \rightarrow 0$ for $r \rightarrow \infty$.

\section{Null circular geodesics of spherically symmetric curved spacetimes}

In the present section we shall follow the analysis presented in $[2,12,18,19]$ in order to determine the radii of the null circular geodesics (closed light rings) which characterize the spherically symmetric self-gravitating ultra-compact objects. We first note that the energy $E$ and the angular momentum $L$ provide two conserved physical parameters along the null geodesics of the static spacetime (5) $[2,12,18,19]$.

In particular, the effective radial potential $[2,12,18,19]^{5}$

$E^{2}-V_{r} \equiv \dot{r}^{2}=\mu\left(\frac{E^{2}}{e^{-2 \delta} \mu}-\frac{L^{2}}{r^{2}}\right)$

determines, through the relations $[2,12,18,19]^{6}$

$V_{r}=E^{2} \quad$ and $\quad V_{r}^{\prime}=0$,

\footnotetext{
$\overline{5}$ Here a dot $\cdot$ denotes a derivative with respect to an affine parameter.

${ }^{6}$ Note that these two functional relations, which determine the null circular geodesics of the spherically symmetric spacetime (5), correspond to $\dot{r}^{2}=\left(\dot{r}^{2}\right)^{\prime}=0[2,12,18]$.
}

the null circular trajectories (light rings) of the static spacetime (5). Substituting Eqs. (8), (9), and (20) into (21), one obtains the characteristic functional relation $[2,12,18,19]$

$\mathcal{R}\left(r=r_{\gamma}\right)=0$,

for the null circular geodesics of the spherically symmetric ultra-compact objects.

\section{An analytical proof of the characteristic relation $\mathcal{C}\left(r_{\gamma}^{\text {in }}\right) \leq \mathcal{C}\left(r_{\gamma}^{\text {out }}\right)$ for horizonless isotropic ultra-compact objects}

The physical properties of spherically symmetric selfgravitating isotropic ultra-compact objects have recently been studied numerically in the interesting work of Novotný, Hladík, and Stuchlík [35] (see also [24]). Intriguingly, it has been explicitly shown in [35] that the horizonless curved spacetimes of these spatially regular compact matter configurations generally possess two light rings $\left\{r_{\gamma}^{\text {in }}, r_{\gamma}^{\text {out }}\right\}$ (see $[26,33]$ for related studies) which are characterized by the dimensionless compactness relation (3).

In the present section we shall use analytical techniques in order to provide a compact proof for the intriguing property $\mathcal{C}\left(r_{\gamma}^{\text {in }}\right)<\mathcal{C}\left(r_{\gamma}^{\text {out }}\right)$ [see Eqs. (3) and (16)] which characterizes the horizonless isotropic ultra-compact objects. We first point out that, taking cognizance of Eqs. (6), (7), (15), and (19), one finds the simple asymptotic relations

$\mathcal{R}(r=0)=2 \quad$ and $\quad \mathcal{R}(r \rightarrow \infty) \rightarrow 2$,

for the dimensionless radial function $\mathcal{R}(r)$. From Eqs. (22) and (23) one deduces that, for horizonless ultra-compact matter configurations with non-degenerate light rings $[26,33]{ }^{7}$ the function $\mathcal{R}(r)$ is characterized by the inequality

$\mathcal{R}(r)<0 \quad$ for $\quad r \in\left(r_{\gamma}^{\text {in }}, r_{\gamma}^{\text {out }}\right)$,

in the radial region between the two light rings of the ultracompact objects.

Substituting the characteristic inequality (24) into Eq. (13) and taking cognizance of the relation (11), one finds that $P(r)$ is a monotonically decreasing function between the two light rings of the horizonless compact object:

$P^{\prime}(r)<0 \quad$ for $\quad r \in\left(r_{\gamma}^{\text {in }}, r_{\gamma}^{\text {out }}\right)$.

\footnotetext{
${ }^{7}$ It is important to note that, as explicitly shown in [33], there are special horizonless matter configurations with degenerate light rings, which are characterized by the relations $\mathcal{R}\left(r=r_{\gamma}\right)=\mathcal{R}^{\prime}\left(r=r_{\gamma}\right)=0$, that may violate the inequality (24). We shall henceforth consider in this section generic $[26,33]$ self-gravitating ultra-compact objects which respect the relation (24).
} 
In particular, from Eqs. (14), (15), (22), and (25), one deduces that the dimensionless function $\mu(r)$ is characterized by the inequality

$\mu\left(r_{\gamma}^{\text {in }}\right) \geq \mu\left(r_{\gamma}^{\text {out }}\right)$

or equivalently [see Eqs. (16) and (18)]

$\mathcal{C}\left(r_{\gamma}^{\text {in }}\right) \leq \mathcal{C}\left(r_{\gamma}^{\text {out }}\right)$

We have therefore provided a simple analytical proof for the numerically observed relation (3) [35] which characterizes the horizonless isotropic ultra-compact objects.

\section{Upper bound on the compactness of the inner light ring of isotropic ultra-compact objects}

The characteristic compactness parameter $\mathcal{C}(r) \equiv m(r) / r$ of the self-gravitating ultra-compact objects can be computed using the numerical procedure described in [35]. Intriguingly, as demonstrated explicitly in [35], the spatially regular horizonless ultra-compact objects may be characterized by inner light rings whose dimensionless compactness parame$\operatorname{ter} \mathcal{C}\left(r_{\gamma}^{\text {in }}\right)$ is well below the lower bound (1) which, as explicitly proved in [25], characterizes the innermost null circular geodesics (light rings) of spherically symmetric asymptotically flat black-hole spacetimes.

In Table 1 we present, for various values of the polytropic index $n$, the numerically computed dimensionless compactness parameter $\mathcal{C}^{\text {numerical }}\left(r_{\gamma}^{\text {in }}\right)$ of the isotropic ultra-compact objects $[35]^{8}[41]$. One finds that $\mathcal{C}\left(r_{\gamma}^{\text {in }} ; n\right)$ is a monotonically decreasing function of the dimensionless polytropic index $n$. Interestingly, we find that the numerical results presented in Table 1 are described extremely well by the simple asymptotic formula (see Table 1)

$\mathcal{C}\left(r_{\gamma}^{\text {in }} ; n\right)=\alpha+\frac{\beta}{n}+O\left(n^{-2}\right)$ with $\alpha=0.2149$ and $\beta=0.1602$.

What we find most interesting is the fact that the horizonless ultra-compact isotropic objects are characterized by the dimensionless asymptotic compactness parameter

$\mathcal{C}\left(r_{\gamma}^{\text {in }} ; n \gg 1\right) \simeq 0.2149<1 / 4$.

\footnotetext{
$\overline{8}$ The numerical results presented in Table 1 correspond to selfgravitating isotropic fluid spheres which are characterized by the limiting pressure-to-density ratio $p_{\mathrm{c}} / \rho_{\mathrm{c}}=n /(n+1)$ at their centers [35]. These matter configurations saturate the upper bound $v_{\mathrm{s}} \leq 1$ on the speed of sound which is imposed by causality requirements [41].
}

Table 1 Ultra-compact polytropic fluid spheres with light rings. We present, for various values of the polytropic index $n$, the numerically computed $[35,41]^{8}$ dimensionless compactness parameter $\mathcal{C}^{\text {numerical }}\left(r_{\gamma}^{\text {in }} ; n\right)$ of the isotropic matter configurations. We also present the corresponding values of the dimensionless compactness parameter $\mathcal{C}^{\text {analytical }}\left(r_{\gamma}^{\text {in }} ; n\right)$ as calculated directly from the simple analytical fit (28). One finds a remarkably good agreement between the numerical results [35] and the analytical formula (28). In particular, one deduces from $(28)$ the characteristic asymptotic value $\mathcal{C}\left(r_{\gamma}^{\text {in }}\right) \rightarrow 0.2149<1 / 4$ for $n \gg 1$

\begin{tabular}{lll}
\hline $\begin{array}{l}\text { Polytropic } \\
\text { index } n\end{array}$ & $\begin{array}{l}\mathcal{C}^{\text {numerical }}\left(r_{\gamma}^{\text {in }}\right) \\
\text { Ref. [35] }\end{array}$ & $\begin{array}{l}\mathcal{C}^{\text {analytical }}\left(r_{\gamma}^{\text {in }}\right) \\
\text { Eq. (28) }\end{array}$ \\
\hline 2.2 & 0.2906 & 0.2877 \\
2.4 & 0.2824 & 0.2817 \\
2.6 & 0.2767 & 0.2765 \\
2.8 & 0.2723 & 0.2721 \\
3.0 & 0.2683 & 0.2683 \\
3.2 & 0.2649 & 0.2650 \\
3.4 & 0.2620 & 0.2620 \\
3.6 & 0.2594 & 0.2594 \\
3.8 & 0.2570 & 0.2571 \\
4.0 & 0.2549 & 0.2550 \\
\hline
\end{tabular}

As emphasized above, one immediately realizes that the asymptotic value (29), which characterizes the spatially regular horizonless matter configurations, is well below the lower bound $\mathcal{C}\left(r_{\gamma}\right) \geq 1 / 3$ [see Eqs. (1) and (16)] which characterizes the corresponding spherically symmetric black-hole spacetimes [25].

In the present section we shall provide an analytical explanation for the numerically inferred asymptotic behavior (29) of the dimensionless compactness parameter. In particular, we shall now derive an upper bound on the compactness parameter $\mathcal{C}\left(r_{\gamma}^{\text {in }} ; n\right)$ of the isotropic ultra-compact objects in the $n \gg 1^{9}$ [37] limit of the polytropic index, which corresponds to the limiting pressure-density relation [see Eq. (2)]

$p=k_{\mathrm{p}} \rho$.

From Eqs. (8), (13), (14), and (15), one finds the gradient relation

$\mathcal{R}^{\prime}\left(r=r_{\gamma}\right)=\frac{2}{r_{\gamma}}\left[1-8 \pi r_{\gamma}^{2}(\rho+p)\right]$

for the isotropic ultra-compact objects. In addition, taking cognizance of Eqs. (22) and (23), one deduces that the inner light ring of a spatially regular ultra-compact horizonless

\footnotetext{
${ }^{9}$ See [37] for the opposite and interesting case of polytropic spheres with $n=0$. As shown in [37], these self-gravitating matter configurations contain only stable null circular geodesics in their interior spacetime regions.
} 
matter configuration is characterized by the relation $\mathcal{R}^{\prime}(r=$ $\left.r_{\gamma}^{\text {in }}\right) \leq 0^{10}$, or equivalently [see Eq. (31)]

$8 \pi r_{\gamma}^{2}(\rho+p) \geq 1$ for $r=r_{\gamma}^{\text {in }}$.

Taking cognizance of Eqs. (15), (22), (30), and (32), one obtains the lower bound

$\mu\left(r_{\gamma}^{\text {in }} ; n \gg 1\right) \geq \frac{2 k_{\mathrm{p}}+1}{3\left(k_{\mathrm{p}}+1\right)}$,

which yields the characteristic upper bound [see Eqs. (16) and (18)]

$\mathcal{C}\left(r_{\gamma}^{\text {in }} ; n \gg 1\right) \leq \frac{k_{\mathrm{p}}+2}{6\left(k_{\mathrm{p}}+1\right)}$,

on the dimensionless compactness parameter which characterizes the inner null circular geodesic (inner light ring) of the isotropic ultra-compact objects. In particular, taking cognizance of Eqs. (11) and (30), one deduces that, in the $n \gg 1$ limit of the polytropic index, the physical parameter $k_{\mathrm{p}}$ is bounded from above by the simple relation $k_{\mathrm{p}} \leq 1$. Substituting the limiting value $k_{\mathrm{p}} \rightarrow 1^{-}$into (34), one obtains the characteristic upper bound ${ }^{11,12}$

$\mathcal{C}\left(r_{\gamma}^{\text {in }} ; n \gg 1, k_{\mathrm{p}} \rightarrow 1^{-}\right)<\frac{1}{4}$.

It is worth emphasizing the fact that the analytically derived upper bound (35) on the dimensionless compactness parameter is consistent with the asymptotic behavior (29) which stems from the numerical studies [35] of the self-gravitating ultra-compact isotropic fluid configurations. In particular, in this section we have provided an explicit analytical proof to the numerically observed intriguing fact

\footnotetext{
${ }^{10}$ It is important to emphasize that in the present section we consider generic spatially regular field configurations with light rings [that is, we consider the case of matter configurations with non-degenerate light rings as well as the special case of matter configurations with degenerate light rings for which $\mathcal{R}^{\prime}\left(r=r_{\gamma}\right)=0$ ].

11 It is worth noting that the upper bound (34) on the physically allowed values of the dimensionless compactness parameter $\mathcal{C}\left(r_{\gamma}^{\text {in }} ; n \gg 1\right)$ is a monotonically decreasing function of the physical parameter $k_{\mathrm{p}}$. For example, substituting the opposite limit $k_{\mathrm{p}} \rightarrow 0^{+}$into (34), one obtains the upper bound $\mathcal{C}\left(r_{\gamma}^{\text {in }} ; n \gg 1, k_{\mathrm{p}} \rightarrow 0^{+}\right)<1 / 3$. The numerical results presented in Table 1 correspond to horizonless ultra-compact isotropic fluid spheres which are characterized by the limiting central ratio $p_{\mathrm{c}} / \rho_{\mathrm{c}}=n /(n+1)$ which is imposed by causality requirements $[35,41]$. In particular, in the $n \gg 1$ regime one finds the limiting behavior $p_{\mathrm{c}} / \rho_{\mathrm{c}} \rightarrow 1^{-}$for the physically acceptable matter configurations, which corresponds to the limiting value $k_{\mathrm{p}} \rightarrow 1^{-}$[see Eq. (30)].

${ }^{12}$ It is important to emphasize that, in the analytical derivation of the upper bound (35), we have not used the inequality (24) which, as explicitly demonstrated in [33], may be violated by special ultra-compact matter configurations with degenerate light rings ${ }^{7}$.
}

that horizonless ultra-compact objects can violate the lower bound (1) which characterizes spherically symmetric blackhole spacetimes. ${ }^{13}$

\section{Summary}

Horizonless spacetimes describing self-gravitating ultracompact matter configurations with closed light rings (null circular geodesics) have recently attracted much attention as possible spatially regular exotic alternatives to canonical black-hole spacetimes (see [26-35] and references therein).

In particular, the physical properties of horizonless ultracompact isotropic fluid spheres with a polytropic equation of state have recently been studied numerically in the physically important work of Novotný, Hladík, and Stuchlík [35]. Interestingly, it has been explicitly shown numerically in [35] that these spherically symmetric spatially regular ultra-compact polytropic matter configurations generally posses two closed light rings (see also $[26,33]$ for related discussions).

In the present paper we have used analytical techniques in order to explore the physical and mathematical properties of the ultra-compact polytropic stars. In particular, it has been explicitly proved that the two light rings of these horizonless matter configurations are characterized by the relation [see Eqs. (16) and (27)]

$\mathcal{C}\left(r_{\gamma}^{\text {in }}\right) \leq \mathcal{C}\left(r_{\gamma}^{\text {out }}\right)$

Interestingly, we have further proved that, while spherically symmetric black-hole spacetimes are characterized by the lower bound $\mathcal{C}\left(r_{\gamma}^{\text {in }}\right) \geq 1 / 3$ [see Eqs. (1) and (16)] [25], the spatially regular horizonless ultra-compact objects are characterized by the opposite dimensionless relation

$\mathcal{C}\left(r_{\gamma}^{\text {in }} ; n \gg 1, k_{\mathrm{p}} \rightarrow 1^{-}\right)<\frac{1}{4}$.

\footnotetext{
${ }^{13}$ It is worth emphasizing that the dimensionless lower bound (1) was derived for spherically symmetric hairy black-hole spacetimes with matter fields which are characterized by an energy-momentum tensor with a negative trace [25,39]. Interestingly, for the isotropic matter fields that we consider in the present paper, this lower bound can be extended to the regime of hairy black-hole spacetimes with generic values of the energy-momentum trace. To see this, we recall that spherically symmetric black-hole spacetimes are characterized by the relation $\mathcal{R}\left(r_{\mathrm{H}} \leq r \leq r_{\gamma}^{\mathrm{in}}\right) \leq 0$ [25], where $r_{\mathrm{H}}$ is the radius of the outer black-hole horizon. Substituting this inequality into (13) and taking cognizance of (11), one finds the characteristic inequality $P^{\prime}\left(r_{\mathrm{H}} \leq r \leq r_{\gamma}^{\text {in }}\right) \leq 0$. This inequality, together with the fact that black-hole spacetimes with regular horizons are characterized by the relation $p\left(r_{\mathrm{H}}\right)=-\rho\left(r_{\mathrm{H}}\right) \leq 0$ [38], yield the important inequality $P\left(r=r_{\gamma}^{\text {in }}\right) \leq 0$. We therefore conclude that hairy black-hole spacetimes with isotropic matter fields and generic values of the energy-momentum trace are characterized by $\mu\left(r=r_{\gamma}^{\text {in }}\right) \leq 1 / 3$ [see Eqs. (14) and (15)], or equivalently $\mathcal{C}\left(r_{\gamma}^{\text {in }}\right) \geq 1 / 3$ [see Eqs. (16) and (18)].
} 
It is worth noting that the analytically derived upper bound (37) on the characteristic dimensionless compactness parameter $\mathcal{C}\left(r_{\gamma}^{\text {in }}\right)$ is consistent with the numerically inferred asymptotic behavior (29).

Finally, it is interesting to emphasize the fact that the analytical results derived in the present paper provide a simple analytical explanation for the interesting numerical results that have recently presented by Novotný, Hladík, and Stuchlík [35] for the physical properties of the self-gravitating ultra-compact polytropic spheres.

Acknowledgements This research is supported by the Carmel Science Foundation. I would like to thank Yael Oren, Arbel M. Ongo, Ayelet B. Lata, and Alona B. Tea for stimulating discussions.

Open Access This article is distributed under the terms of the Creative Commons Attribution 4.0 International License (http://creativecomm ons.org/licenses/by/4.0/), which permits unrestricted use, distribution, and reproduction in any medium, provided you give appropriate credit to the original author(s) and the source, provide a link to the Creative Commons license, and indicate if changes were made. Funded by SCOAP ${ }^{3}$.

\section{References}

1. J.M. Bardeen, W.H. Press, S.A. Teukolsky, Astrophys. J. 178, 347 (1972)

2. S. Chandrasekhar, The Mathematical Theory of Black Holes (Oxford University Press, New York, 1983)

3. S.L. Shapiro, S.A. Teukolsky, Black Holes, White Dwarfs and Neutron Stars: The Physics of Compact Objects, 1st edn. (WileyInterscience, Hoboken, 1983)

4. M.A. Podurets, Astr. Zh. 41, 1090 (1964). [English translation in Sovet Astr.-AJ 8, 868 (1965)]

5. W.L. Ames, K.S. Thorne, Astrophys. J. 151, 659 (1968)

6. I.Z. Stefanov, S.S. Yazadjiev, G.G. Gyulchev, Phys. Rev. Lett. 104, 251103 (2010)
7. C.J. Goebel, Astrophys. J. 172, L95 (1972)

8. B. Mashhoon, Phys. Rev. D 31, 290 (1985)

9. S.R. Dolan, Phys. Rev. D 82, 104003 (2010)

10. Y. Dećanini, A. Folacci, B. Raffaelli, Phys. Rev. D 81, 104039 (2010)

11. Y. Dećanini, A. Folacci, B. Raffaelli, Phys. Rev. D 84, 084035 (2011)

12. V. Cardoso, A.S. Miranda, E. Berti, H. Witek, V.T. Zanchin, Phys. Rev. D 79, 064016 (2009)

13. S. Hod, Phys. Rev. D 80, 064004 (2009). arXiv:0909.0314

14. S. Hod, Phys. Rev. D 78, 084035 (2008). arXiv:0811.3806

15. S. Hod, Phys. Rev. D 75, 064013 (2007). arXiv:gr-qc/0611004

16. S. Hod, Class. Quant. Grav. 24, 4235 (2007). arXiv:0705.2306

17. S. Hod, Phys. Lett. B 715, 348 (2012). arXiv:1207.5282

18. S. Hod, Phys. Rev. D 84, 124030 (2011). arXiv: 1112.3286

19. S. Hod, Phys. Rev. D 84, 104024 (2011). arXiv: 1201.0068

20. S. Hod, Phys. Lett. B 718, 1552 (2013). arXiv:1210.2486

21. S. Hod, Phys. Lett. B 751, 177 (2015). arXiv:1707.06246

22. S. Hod, Class. Quant. Grav. 33, 114001 (2016). arXiv: 1705.08905

23. R.A. Konoplya, Z. Stuchlík, Phys. Lett. B 771, 597 (2017)

24. Z. Stuchlík, J. Schee, B. Toshmatov, J. Hladik, J. Novotný, JCAP 06, 056 (2017)

25. S. Hod, Phys. Lett. B 727, 345 (2013). arXiv: 1701.06587

26. P.V.P. Cunha, E. Berti, C.A.R. Herdeiro, arXiv:1708.04211

27. V. Cardoso, L.C.B. Crispino, C.F.B. Macedo, H. Okawa, P. Pani, Phys. Rev. D 90, 044069 (2014)

28. S. Hod, Phys. Lett. B 739, 383 (2014). arXiv:1412.3808

29. E. Maggio, P. Pani, V. Ferrari, arXiv: 1703.03696

30. S. Hod, J. High Energy Phys. 06, 132 (2017). arXiv: 1704.05856

31. S. Hod, Phys. Lett. B 770, 186 (2017)

32. S. Hod, Phys. Lett. B 774, 582 (2017). arXiv:1708.09399

33. S. Hod, arXiv: 1710.00836

34. J. Keir, Class. Quant. Grav. 33, 135009 (2016)

35. J. Novotný, J. Hladík, Z. Stuchlík, Phys. Rev. D 95, 043009 (2017)

36. G.P. Horedt, Polytropes, Applications in Astrophysics and Related Fields (Kluwer Academic Publishers, Dordrecht, 2004)

37. Z. Stuchlík, S. Hledík, J. Novotný, Phys. Rev. D 94, 103513 (2016)

38. A.E. Mayo, J.D. Bekenstein, Phys. Rev. D 54, 5059 (1996)

39. H. Bondi, Mon. Not. Roy. Astr. Soc. 259, 365 (1992)

40. S.W. Hawking, G.F. Ellis, The Large Scale Structure of Spacetime (Cambridge University Press, Cambridge, 1973)

41. R.F. Tooper, Astrophys. J. 140, 434 (1964) 